\title{
Gravity Transform for Input Conditioning in Brain Machine Interfaces
}

\author{
António R. C. Paiva, José C. Príncipe and Justin C. Sanchez
}

\begin{abstract}
Gravity transform measures cooperative neural activity being utilized for the analysis of neural assemblies. In this paper we verify the applicability of the gravity transform to specify components of neural assemblies, which could be combined, leading ultimately to a reduction of the input dimensionality in brain-machine interface models. Our analysis was performed on data collected from rats performing a lever pressing task. We compare the results from the gravity transform analysis with the assignment obtained through a sensitivity analysis applied on a linear optimal filter.
\end{abstract}

\section{INTRODUCTION}

B RAIN Machine Interfaces (BMIs) use recordings of the activity of an ensemble of cortical neurons to control motor neuroprosthetic systems. Due to the localized nature of the activity of single neurons, BMIs have much higher resolution and specificity than neuroprosthetic systems relying on other neural recordings. The downside of this approach however is the sparseness of the neural activity, reason why a much higher number of signals needs to be collected, conditioned, and analyzed. This translates in new challenges for modeling. The natural approach to cope with the dimensionality problem is to select or group inputs based on some relevant measure.

Previous methods for input dimensionality in BMIs have done so in a supervised manner, either through architectures with higher descriptive power but with smaller number of parameters or methods that estimate the importance of the input components for pruning. An example of the former case is the work by Sanchez [1] in which a recurrent neural network (RNN) was used. The fact that the time-structure in this architecture becomes embedded in the weights of the recurrent connections reduced the total number of weights to estimate. Nevertheless, this is achieved through more complex training. After training, the model output sensitivity to each input together with the variance of the input data provides a simple yet effective relevance measure of the input. This was used in either linear or nonlinear models, such as FIR or RNN models to prune the trained model [1]-[3]. Kim [2] also proposed a online method, appropriately named Least Angle Regression, for this input selection based on the

This work was supported in part by DARPA under grant ONR450595112, the National Science Foundation under Grant \#CNS-0540304 and the Children's Miracle Network. The work of A. R. C. Paiva was supported by Fundação para a Ciência e a Tecnologia under grant SFRH/BD/18217/2004.

António R. C. Paiva and José C. Príncipe are with the Computational NeuroEngineering Laboratory, Electrical and Computer Engineering Department, University of Florida, Gainesville, FL 32611 USA \{arpaiva, principe\}@cnel.ufl.edu

Justin C. Sanchez is with the Department of Pediatrics, Division of Neurology, and McKnight Brain Institute at the University of Florida, Gainesville, FL 32611, USA justin@ cnel. ufl. edu angle between the input and the model output. Unfortunately the problem was formulated under a prediction framework and hence the desired response is continually required. Other methods include the use of principal component analysis and partial least squares [4], [5], yet the selection of the projections are performed using a significance test, since frequently the most descriptive of the behavior was not the one with higher variance.

Our aim in this study, however, is towards a method for input selection/conditioning without any need for the desired signal. This unsupervised approach is particularly interesting for paralyzed patients who are using BMIs where a desired signal is unavailable. For training the BMI it is critical to identify cell assemblies that have synchronous modulation. For this task we selected the gravity transform [6]. When compared to other tools commonly used for analysis of neural ensembles in neuroscience, such as the cross-correlation, joint peri-stimulus time histogram (JPSTH) [7] and partial direct coherence (PDC) [8], the gravity transform provides a more convenient view of the synchrony interactions among all neurons pairs. Actually, for the dataset used, where a total of 276 neuron pairwise combinations exist, an analysis with the other methods would be simply overwhelming. Furthermore, it is claimed that under ideal conditions the gravity transform could provide an overview of instantaneous synchrony relationships [6].

\section{Materials And Methods}

\section{A. Data}

Multielectrode array recordings were collected from male Sprague-Dauley rats performing a go-no go lever pressing task. Array configurations of two rows of eight tungsten electrodes $(2 \times 8)$, spaced $250 \mu \mathrm{m}$ between columns and $500 \mu \mathrm{m}$ between rows were chronically implanted in the forelimb region of $\mathrm{M} 1+1.0 \mathrm{~mm}$ anterior, $2.5 \mathrm{~mm}$ lateral of bregma). Neuronal activity was collected with a TuckerDavis recording rig with sampling frequency of $24414.1 \mathrm{~Hz}$ and digitized to 16 bits of resolution. Before further processing the neuronal potentials were bandpass filtered between $300 \mathrm{~Hz}$ to $7 \mathrm{kHz}$ to extract the action potentials. Subsequently the data was spike detected and spike sorted using thresholding and template matching [9]. The lever presses were recorded simultaneously with the neural activity and were downsampled to $381.5 \mathrm{~Hz}$.

In this dataset, 29 channels had action potentials above the noise floor from which, after spike sorting, we obtained 44 spike trains from single neurons, 24 from the left hemisphere of the brain and 20 from the right hemisphere. For this work we used only the first 24 spike trains corresponding to the 
activity recorded from the left hemisphere of the brain, to ease the analysis and since they were more relevant for the modeling task than the ones from the right hemisphere.

\section{B. Gravity Transform}

The concept of the gravity transform (also named gravity clustering) was first introduced as a neuroscience analysis tool by Gerstein et al. [6]. The idea was motivated by the need to search for synchronous neurons in neural assemblies but with smaller computational requirements and ease of interpretation than the cross-correlation. In the gravity transform the distance computed over time between each neuron pair in a hypothetical hyperspace is a descriptor of the synchrony between the two neurons and hence their correlation. In general terms, the transform considers each neuron as a particle ${ }^{1}$ in a $N$-dimensional space, where $N$ is the number of neurons in the analysis. In order to define a gravity interaction between particles we need to first define a "mass" or "charge" from which the interaction force is expressed. Since in this case we are interested in neural assemblies defined in terms of synchronous firing we can define the charge based on the spike times. Then neurons firing synchronously will charge at the same time and attract each other. Overall, we can see the distance in the space between synchronous neurons to reduce as time evolves.

In more detail, the algorithm proceeds as follows. Consider the algorithm is applied to $N$ neurons. First, the particles are placed in a $N$-dimensional space so that they are equidistant. Writing the position of the $i$-th neuron in this space as the coordinate vector, $\mathbf{x}_{i}=\left[x_{i 1}, x_{i 2}, \ldots, x_{i N}\right]^{T}$, this step can be done very easily by setting the $i$-th coordinate component equal to 0.5 and all other entries to zero. Spatially, this corresponds to position each neuron at the vertex of an $N$ dimensional hypercube. With these values the initial distance between any two neurons is one. Other values can be used simply by scaling the coordinate vectors.

As we stated before, the underlying principle of the definition of neural assemblies is the synchrony between neurons. But exact synchrony in the spike times is extremely difficult, therefore a exponential kernel is used to extend the effect of a spike over time. The time constant $\tau$ of this exponential controls the decay of the charge over time

$$
q(t)=q(0) e^{-t / \tau}
$$

where $q(0)$, is the charge added by each spike.

Now that we have defined how the charge will be regulated, we can use the force to determine the neuron coordinate update rule. However, unlike physical systems, the force is independent from distance between particles and affects linearly the velocity rather than the acceleration. The former is because the distance here is a conceptual formulation and therefore has no meaning; the latter because it simplifies the problem to a first order differential equation. Thus, the

\footnotetext{
${ }^{1}$ We will use the term neuron and particle interchangeably.
}

velocity of the $i$-th particle in space is given by

$$
\frac{d \mathbf{x}_{i}(t)}{d t}=\eta q_{i}(t) \sum_{\forall j \neq i} q_{j}(t) \frac{\left(\mathbf{x}_{j}-\mathbf{x}_{i}\right)}{\left\|\mathbf{x}_{j}-\mathbf{x}_{i}\right\|},
$$

where $q_{i}(t)$ is the charge of the $i$-th particle and $\eta$ is the mobility of the particles ("learning rate"). The $\left(\mathbf{x}_{j}-\right.$ $\left.\mathbf{x}_{i}\right) /\left\|\mathbf{x}_{j}-\mathbf{x}_{i}\right\|$ term is simply a unit length vector pointing in the direction of the line that passes through both particles. So, the velocity of the particle is the direction and proportional to the sum of the force vectors between the neuron and all others. Using Euler integration with step $d t$, we obtain the final particle coordinate update equation

$$
\mathbf{x}_{i}(t+d t)=\mathbf{x}_{i}(t)+\eta q_{i}(t) \sum_{\forall j \neq i} q_{j}(t) \frac{\left(\mathbf{x}_{j}-\mathbf{x}_{i}\right)}{\left\|\mathbf{x}_{j}-\mathbf{x}_{i}\right\|} d t
$$

There exist two problems in the previous formulation however: the dependence on the firing rate and the always attracting force. Both can be solved through the normalization of the charge. To the first we want the mean charge to be equal to all neurons. To prevent attraction due to correlation by chance we simply make the mean charge zero. So that for the above definitions we used

$$
q_{i}(t)=\frac{1}{\lambda_{i}} e^{-t / \tau}-\tau
$$

Beyond this we also implemented the 1-D interaction suggested by Lindsey and Gerstein [10] which can be implemented very simply by using the Manhattan distance instead of Euclidean distance. With our definition of the original coordinates for each point we will have an initial distance of one for all neuron pairs.

\section{Modelling and output sensitivity analysis}

For modeling of the transfer function between spike trains and hand movements we used a multiple input linear filter [11]-[13] trained using the Wiener solution. For effective lever status prediction we can apply a hard threshold to the filter output. This architecture has been used extensively in BMIs [13] and was chosen based on the ease of training and in performing a sensitivity analysis. First the firing rate of the spike trains in $100 \mathrm{~ms}$ non-overlapping time windows $(10 \mathrm{~Hz})$ was computed and the level press signal re-sampled to the same rate. At the input layer each input is embedded in a higher dimensional space through a 10-tap delay line [12]. These signals are then combined in a weighted sum with weights given by the Wiener-Hopf equation,

$$
\mathbf{W}=\mathbf{R}^{-1} \mathbf{P}
$$

where $\mathbf{R}$ is the correlation matrix of the inputs and $\mathbf{P}$ the correlation between inputs and desired.

After training the model the weights also provide information on the relevance of the neurons for the modeling of the output [1], [14]. The "sensitivity" 2 of the desired response to the $i$-th neuron can be obtained as the average sensitivity

\footnotetext{
${ }^{2}$ Notice the difference in this definition of the sensitivity since the descriptive information of the inputs needs to be taken into account, unlike the more common formulation which is input independent.
} 

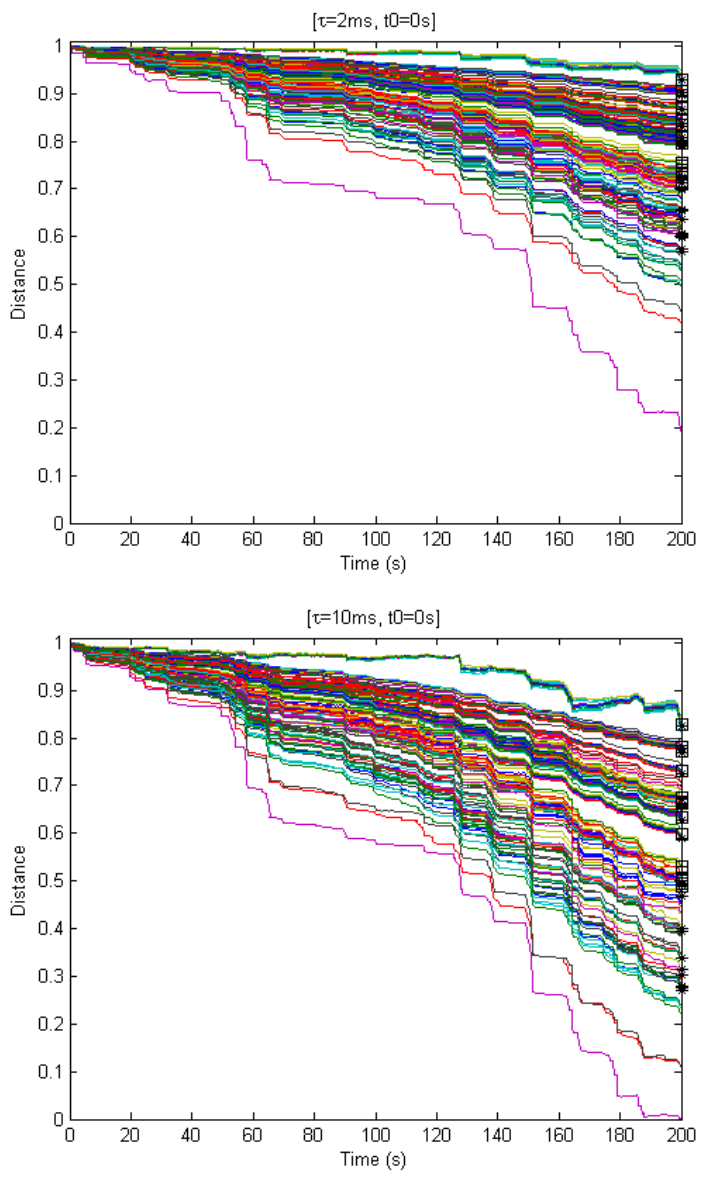

(a)

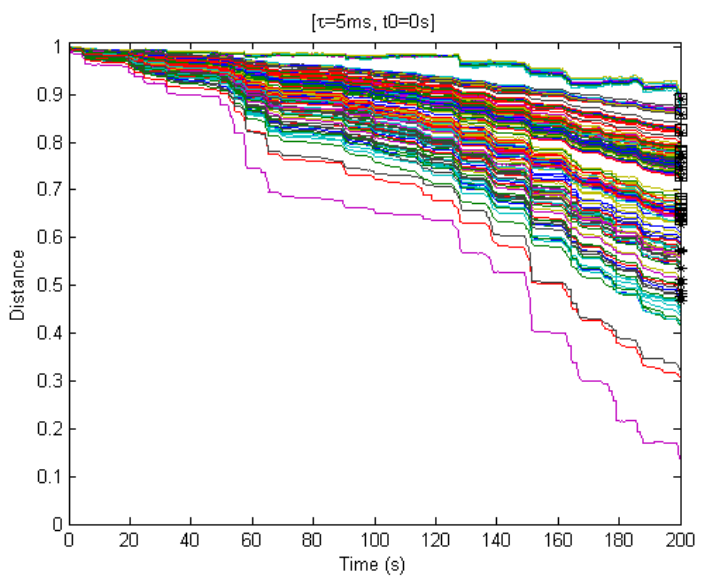

(b)

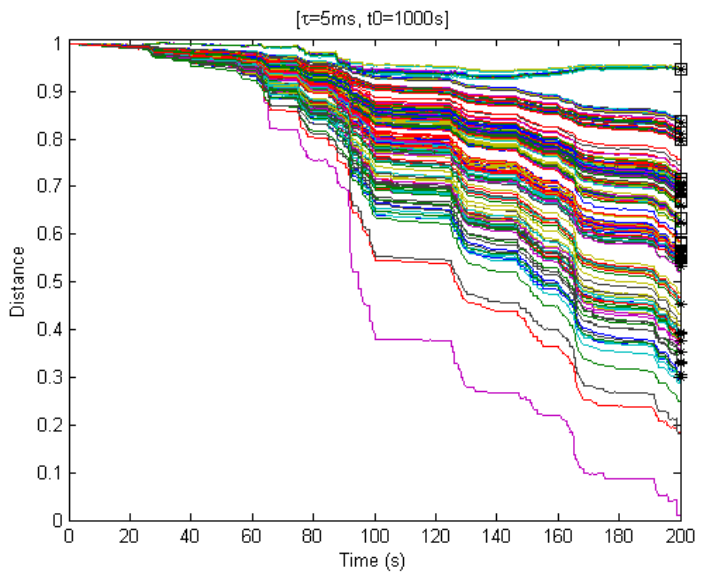

Fig. 1. Distance over time between all neuron pairs as obtained by the gravity transform. Plots (a)-(c) show the gravity transform results applied on the first 200 seconds of data with decay time constant $2 \mathrm{~ms}, 5 \mathrm{~ms}$ and $10 \mathrm{~ms}$, respectively. Plot (d) shows similar results on a different segment of $200 \mathrm{~s}$ of data with a decay time constant of $5 \mathrm{~ms}$. The mobility parameter is different between plots. The $\left(^{*}\right)$ and $(\square)$ marks the lines corresponding to interactions with neurons 7 and 23 , respectively, to which the output is the most sensitive.

over the taps, i.e., $S_{i}=\frac{1}{10} \sum_{j=0}^{9} \mathbf{W}_{10(i-1)+j} \sigma_{i}$, where $\sigma_{i}$ the standard deviation of the binned data for the $i$ th neuron.

\section{DATA ANALYSIS}

As stated previously, our first aim here was to use the gravity transform to search for neural assemblies. With this in mind, we applied the gravity transform to segments of 200 seconds of our dataset. First the algorithm was applied to the first 200 seconds of data for three different exponential decay time constants. Figure 1(a)-(c) shows these results, for $\tau$ equal to $2 \mathrm{~ms}, 5 \mathrm{~ms}$ and $10 \mathrm{~ms}$, respectively. These values are in the range of the values used throughout the literature [6], [10], [15]. The particles mobility was chosen to be $2.0,0.8$ and 0.4 , respectively, so that it would increase the spread of distances at the end of convergence while preventing these to reach zero. In all the cases considered here the time integration step was $1 \mathrm{~ms}$ and the initial distance between neurons set to unity. Figure 1(d) shows the same case as Fig. 1(b), with $\tau=2 \mathrm{~ms}$ and particle mobility 0.8 , but on a different segment of data starting at $1000 \mathrm{~s}$, relative to the beginning of the dataset. In all four plots the three faster converging neuron pairs correspond to $(3,19)$, $(3,24)$ and $(19,24)$. The remaining fast decreasing lines are mostly interaction between one of the neurons from the above pairs and other neurons. The lines corresponding to the two most significant neurons (Fig. 3) are marked. Despite the obvious differences between the first three plots due to charge variability [15] and due to a different data segment, these plots show that the algorithm fundamental result is essentially the same in all cases, and hence our analysis is independent of the decay time constant chosen and data segment.

Considering the possibility of time delays between neurons considerably greater than the decay time constant, to which the gravity transform algorithm used would be insensitive [10], we investigated as well the cross-correlation for all neuron pairs calculated over the whole dataset with $1 \mathrm{~ms}$ resolution between $-75 \mathrm{~ms}$ and $75 \mathrm{~ms}$. The result is shown in Fig. 2. As can be observed the cross-correlation of all neuron pairs is centered at lag zero.

Based on the information extracted from the distance plots obtained with the gravity transform we combined the sets of cooperating neurons to reduce the input dimensionality of the models. Yet, we found ourselves instead with the observation that some level of synchrony is present among all pairs of neurons (Fig. 1). Moreover, Fig. 2 reveals that this synchrony 


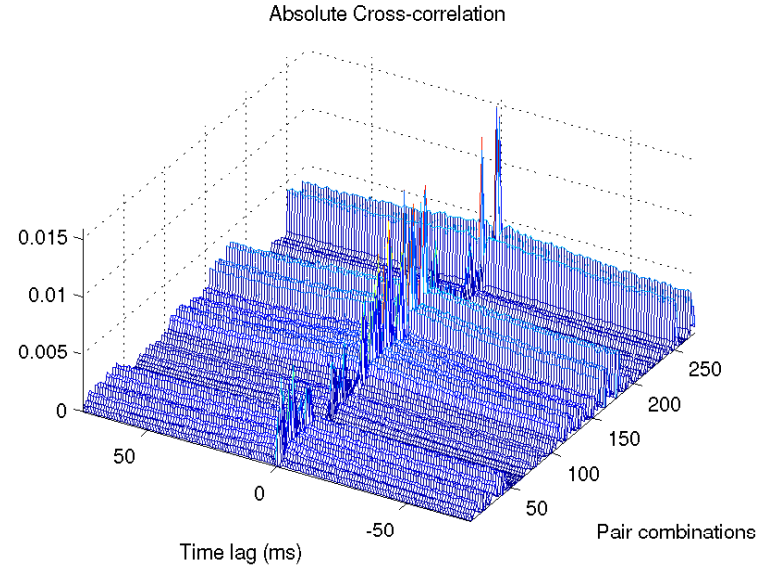

Fig. 2. Cross-correlation plot for all neuron pairs.

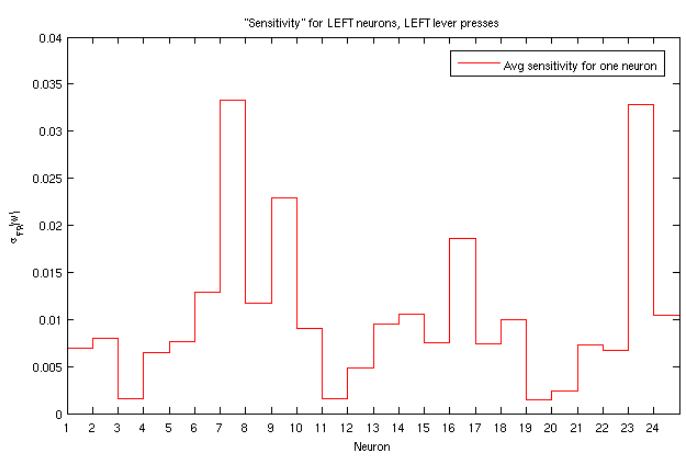

Fig. 3. Sensitivity of the output to each of the 24 neurons averaged across lags.

is well localized in time. What most negatively affects our hypothesis however is the almost uniform distribution of the distances between particles over the range, including for the neurons found most significant for modeling. This unlike other results presented for in vivo data [10], and in a way quite different from what is obtained with simulated datasets. Furthermore, Fig. 3 shows that the relevance to BMI modeling of the neurons corresponding to the most correlated activity is very small.

\section{DISCUSSION}

Data analysis to improve modeling capabilities is an important component of BMI research. In this paper we analyzed the applicability of an (unsupervised) analysis tool developed in neuroscience for the study of neural assemblies with the intention of performing data dimensionality reduction based on this analysis. Our results reveal however that neuron ensembles are not easily defined. Although we were unable to reach the final goal, the results verify the interconnectivity of the neurons at the level of the MI cortex area. Moreover, considering the electrodes grid physical dimensions, the results show that this interconnectivity goes beyond the range of an electrode [16]. The fact that the crosscorrelation between the neuron pairs is centered at zero lag further supports our analysis in terms of time synchrony.
We were also able to find that the underlying metric of the gravity transform is not appropriate for our modeling analysis. When using unsupervised analysis tools caution is required to ensure an effective representation of the information we ultimately interested in. As our sensitivity analysis verifies, in a somewhat extreme manner, the long term synchrony of neural activity between neurons does not translate necessarily in importance to the output modeling. This is not to say that unsupervised methods are not possible or appropriate for this task, rather we wish only to remark here what seems to us the inadequacy of the gravity transform for modeling input dimensionality reduction.

\section{REFERENCES}

[1] J. C. Sanchez, "From cortical neural spike trains to behavior: modeling and analysis," Ph.D. dissertation, University of Florida, May 2004.

[2] S.-P. Kim, "Design and analysis of optimal decoding models for brainmachine interfaces," Ph.D. dissertation, University of Florida, May 2005.

[3] J. C. Sanchez, J. M. Carmena, M. A. Lebedev, M. A. L. Nicolelis, J. G. Harris, and J. C. Principe, "Ascertaining the importance of neurons to develop better brain-machine interfaces," IEEE Trans. on Biomedical Engineering, vol. 51, no. 6, pp. 943-953, June 2004.

[4] J. Hu, J. Si, B. P. Olson, and J. He, "Feature detection in motor cortical spikes by principal component analysis," IEEE Trans. on Neural Systems and Rehab. Eng., vol. 13, no. 3, pp. 256-262, Sept. 2005.

[5] J. Hu, J. Si, B. P. Olson, R. S. Clement, and J. He, "Decoding motor cortical spike trains for brain machine interface applications," in Proc. of the 25th Annual Int. Conf. the IEEE EMBS, vol. 3, Cancun, Mexico, Sept. 2003, pp. 2071-2074.

[6] G. L. Gerstein, D. H. Perkel, and J. E. Dayhoff, "Cooperative firing activity in simultaneously recorded populations of neurons: detection and measurement," Journal of Neuroscience, vol. 5, no. 4, pp. 881$889,1985$.

[7] A. M. Aertsen, G. L. Gerstein, M. K. Habib, and G. Palm, "Dynamics of neuronal firing correlation: modulation of "effective connectivity"," Journal of Neurophysiology, vol. 61, no. 5, pp. 900-917, 1989.

[8] K. Sameshima and L. A. Baccalá, "Using partial directed coherence to describe neuronal ensemble interactions," Journal of Neuroscience Methods, vol. 94, no. 1, pp. 93-103, Dec. 1999.

[9] M. S. Lewicki, "A review of methods for spike sorting: the detection and classification of neural action potentials," Network: Computation in Neural Systems, vol. 9, no. 4, pp. R53-R78, 1998.

[10] B. G. Lindsey and G. L. Gerstein, "Two enhancements of the gravity algorithm for multiple spike train analysis," Journal of Neuroscience Methods, vol. 150, no. 1, pp. 116-127, 2006.

[11] S. Haykin, Adaptive Filter Processing, 4th ed. Prentice-Hall, 2002.

[12] S. P. Kim, J. C. Sanchez, Y. N. Rao, D. Erdogmus, J. M. Carmena, M. A. Lebedev, M. A. L. Nicolelis, and J. C. Principe, "A comparison of optimal mimo linear and nonlinear models for brain-machine interfaces," Journal of Neural Engineering, 2006, in press.

[13] J. Wessberg, C. R. Stambaugh, J. D. Kralik, P. D. Beck, M. Laubach, J. K. Chapin, J. Kim, S. J. Biggs, M. A. Srinivasan, and M. A. L. Nicolelis, "Real-time prediction of hand trajectory by ensembles of cortical neurons in primates," Nature, vol. 408, no. 6810, pp. 361365, Nov. 2000.

[14] J. C. Sanchez, D. Erdogmus, Y. Rao, S.-P. Kim, M. Nicolelis, J. Wessberg, and J. C. Principe, "Interpreting neural activity through linear and nonlinear models for brain machine interfaces," in Proc. of the 25th Annual Int. Conf. of IEEE EMBS, Cancun, Mexico, Sept. 2003, pp. 2160-2163.

[15] S. N. Baker and G. L. Gerstein, "Improvements to the sensitivity of gravitational clustering for multiple neuron recordings," Neural Computation, vol. 23, no. 11, pp. 2597-2620, 2000

[16] G. Buzsáki, "Large-scale recording of neuronal ensembles," Nature Neuroscience, vol. 7, no. 5, pp. 446-451, May 2004. 